\title{
ARTICLE
}

\section{Diagnostic accuracy of single channel cystometry for neurogenic bladder diagnosis following spinal cord injury: a pilot study}

\author{
Akmal Hafizah Zamli ${ }^{1}$, Kavitha Ratnalingam ${ }^{1}$, Yusma Asni Yusmido ${ }^{2}$ and Kuo Ghee Ong ${ }^{3}$
}

INTRODUCTION: This is a cross-sectional study of 1 year duration (August 2013 to August 2014). The objective of the study was to investigate the diagnostic accuracy of single channel cystometry (SCC) for confirmation of neurogenic bladder following spinal cord injury.

MATERIALS AND METHODS: The study was conducted in both out-patient and in-patient services of Department of Rehabilitation Medicine, Hospital Sungai Buloh, Malaysia. Subjects in the study include sixteen patients with a clinical diagnosis of neurogenic bladder following spinal cord injury aged between 15 and 62 years. Patients with a clinical diagnosis of neurogenic bladder were subjected to cystometric evaluation using SCC in our hospital. Confirmation of the diagnosis was made by urodynamic study (UDS) in another hospital. SCC procedure involved manual intra-vesical pressure assessment using a 12F Nelaton catheter. Cystometric parameter measurement taken in this study was detrusor pressure $\left(\mathrm{cm} \mathrm{H}_{2} \mathrm{O}\right)$ done at regular intervals from baseline, throughout bladder filling phase and voiding/leaking phase. The relationship between detrusor pressure to bladder volume from initial bladder filling until voiding or leaking phase was recorded, analyzed and graph plotted. Maximum detrusor pressure $\left(\mathrm{cm} \mathrm{H}_{2} \mathrm{O}\right)$ during bladder filling, voiding or leaking and the maximum cystometric capacity (mls) was recorded.

RESULTS: SCC was found to have $100 \%$ sensitivity, 50\% specificity, $93.33 \%$ positive predictive value and 100\% negative predictive value for neurogenic bladder diagnosis. Only 55.55\% patients with SCC suspicion of detrusor sphincter dyssynergia (DSD) had comparable UDS findings.

DISCUSSION: The use of SCC may be considered for objective confirmation of neurogenic bladder diagnosis following spinal cord lesion in centers with limited access to UDS. However, we are not able to establish its clinical application for confirmation of DSD.

Spinal Cord Series and Cases (2017) 3, 16044; doi:10.1038/scsandc.2016.44; published online 4 May 2017

\section{INTRODUCTION}

A significant number of people with spinal cord injury $(\mathrm{SCl})$ are left with the debilitating condition of neurogenic bladder dysfunction (NBD). The actual prevalence of NBD in patients with $\mathrm{SCl}$ is not known; however, $81 \%$ of such patients were estimated to report some degree of impaired bladder function within 1 year of injury. ${ }^{1}$ From a population of patients with suprasacral injuries, Jeong et al. ${ }^{2}$ found that as many as $93-100 \%$ of patients with complete injury and $93 \%$ of patients with incomplete injury demonstrated urodynamic evidence of neurogenic detrusor overactivity, with or without detrusor sphincter dyssynergia (DSD). Therefore it is imperative that this condition is managed well to prevent further medical complications such as urinary tract infection, vesicoureteral reflux, hydronephrosis and calculi formation. In addition, from the patient's perspective, it facilitates social re-integration and improves quality of life. ${ }^{1,2}$

The International Spinal Cord Medicine Consortium recognizes urodynamic study (UDS) as the gold standard for the diagnosis and evaluation of NBD. It is also the tool of choice to guide the management of bladder dysfunction in patients with $\mathrm{SCl}$. The multi-channel probes in UDS are able to isolate detrusor pressure, which is the key determinant of bladder safety. 3,4
Since the emergence of UDS, other methods of intra-vesical pressure measurement are no longer used. This includes the single channel cystometry (SCC). In most developed countries UDS is widely available; however, because of the high cost of both the machine and consumables required, its availability remains limited in less developed countries, including Malaysia. This coupled with the high patient load in public hospitals causes a long waiting time for the assessment and management of NBD with UDS. In view of this, we practice SCC for the early diagnosis and management of NBD.

The significance of this study rests on the fact that while many previous studies have compared the diagnostic accuracy of SCC versus UDS in non-NBD, ${ }^{5-8}$ none of these have studied bladder dysfunction of neurogenic etiology, much less in a population of patients with NBD due to SCl. As such this is the focus of our study.

\section{MATERIALS AND METHODS}

This is a cross-sectional study done over 1 year, from August 2013 to August 2014. Ethical approval was by the local institutional board with reference number NMRR-15-2264-28028. The study was conducted in both in-patient and out-patient rehabilitation facilities of the Department of Rehabilitation Medicine, Hospital Sungai Buloh, Malaysia.

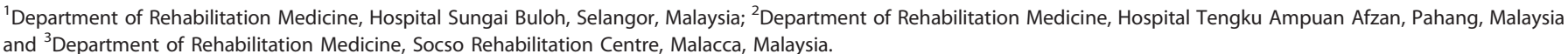




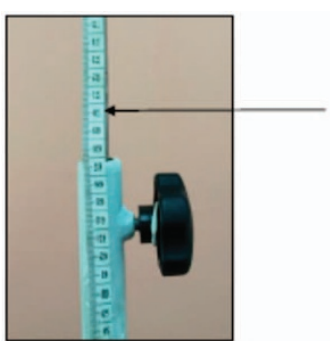

1. The measuring tape was vertically attached onto the drip stand with centimetre markings facing outwards.

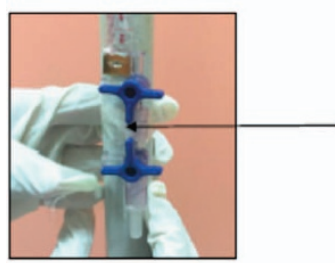

2. Two three way valves were vertically attached onto the drip stand at the zero mark of the measuring tape. The upper one served as the main switch.

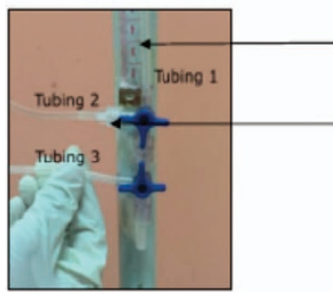

3. Tubing 1 was vertically attached onto the drip stand with its top open.

4. Tubing 2 was connected to the Normal saline drip for bladder infusion.

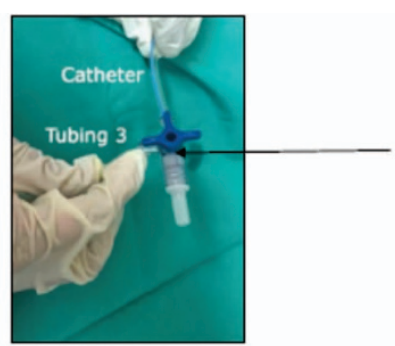

5. Tubing 3 was connected to the Nelaton catheter.

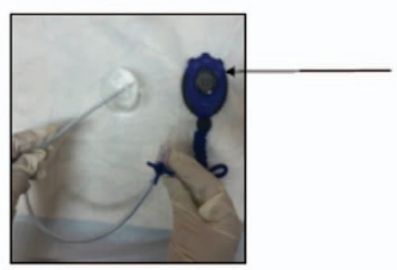

6. Infusion rate was manually determined.

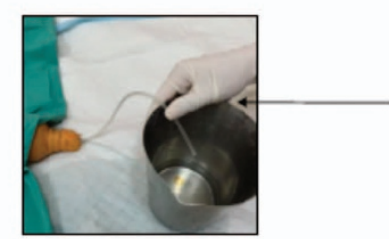

Figure 1. The main steps in the setting up of single channel cystometry (SCC) used in this study.

Subjects enrolled had to meet the following inclusion criteria: (i) confirmed $\mathrm{SCl}$ of either traumatic or non-traumatic etiology; (ii) a clinical diagnosis of neurogenic bladder with persistent lower urinary tract symptoms refractory to non-pharmacological intervention; (iii) patients considered for initiation of pharmacological treatment for symptomatic control of bladder symptoms; and (iv) consented to the study. Subjects excluded were those with (i) active urinary tract infection, (ii) anatomical or structural deformities of the lower urinary tract and (iii) non-consenting patients.

All methods, definitions and units used in this study conformed to the standards recommended by the International Continence Society, except where specifically stated. ${ }^{9}$ Out of a total of 32 patients with a clinical diagnosis of neurogenic bladder, 16 fulfilled the study criteria. The nonpharmacological intervention consisted of fluid management, pelvic floor exercises and intermittent catheterization either by self or caregiver. All patients in this study were subjected to a detailed neurological assessment including per rectal examination prior to SCC evaluation in our hospital. Patients were then subjected to a confirmatory UDS assessment in another hospital.

\section{Single channel cystometry procedure}

We adapted the SCC protocol published by Wyndaele et al..$^{10}$ to suit our local resources. The main steps involved in setting up the SCC unit are illustrated in Figure 1. Equipment included a drip stand with a bottle of normal saline $0.9 \%(500 \mathrm{ml})$ attached via two 3-way connectors to a transparent plastic tubing vertically taped to the drip stand with its top end open. A measuring tape with its lower end at $0 \mathrm{~cm}$ reading and upper end at $120 \mathrm{~cm}$ reading was taped alongside the plastic tubing. A second plastic tubing was connected to the lower 3-way connector which was then attached to a size $12 \mathrm{~F}$ Nelaton catheter via a third 3-way connector. The $0 \mathrm{~cm}$ reading was adjusted to the same level as the estimated bladder level by adjusting the bed height. A graph paper was prepared with its $x$ axis marked in mls (bladder volume) and its $y$ axis marked from 0 to $120 \mathrm{~cm} \mathrm{H}$ O (intra-vesical pressure $\left(P_{\text {ves }}\right)$ ).

When the SCC unit was ready, filling speed was determined by adjusting the roller valve until the desired flow rate of $20-30 \mathrm{mls}$ per minute was achieved. The roller valve was then secured so that the flow rate remained constant. The upper 3-way connector was used as the main control throughout the procedure. The bladder was emptied prior to the SCC procedure using a Nelaton catheter. Intra-vesical catheter placement was confirmed by observing the meniscus movement when the patient was instructed to cough. Patients were instructed to avoid movement and to maintain normal breathing throughout the procedure to minimize the influence of intra-abdominal pressure $\left(P_{\text {abd }}\right.$ on intra-vesical pressure $\left(P_{\text {ves }}\right)$. Thus, for the purpose of this study, intra-vesical pressure $\left(P_{\text {ves }}\right)$ is equivalent to detrusor pressure $\left(P_{\text {det }}\right)$.

The opening $P_{\text {ves }}$ was measured and infusion started at a predetermined rate. $P_{\text {ves }}$ was recorded at intervals of $1 \mathrm{~min}$. Bladder volume at each minute was determined by multiplying time to filling speed. Additional measurements of $P_{\text {ves }}$ and bladder volume were recorded at first, normal and strong desire to void as well as sensations of urgency. Bladder filling was stopped after $500 \mathrm{mls}$ infusion or if the patients developed urgency, leaking, symptoms of autonomic dysreflexia or a rise of $P_{\text {ves }}$ to $>80 \mathrm{~cm} \mathrm{H}_{2} \mathrm{O}$. If the patient voided voluntarily, the voided volume as well as post-void residual volume were measured. However, if no voluntary voiding occurred, the bladder was emptied and its volume recorded. These steps were to ensure that the total infused volume was consistent with the estimated bladder volume.

\section{Urodynamic study procedure}

The UDS procedure was performed in a different hospital using the Mediwatch urodynamic device. Intra-vesical $\left(P_{\text {ves }}\right)$ and intra-abdominal pressures $\left(P_{\mathrm{abd}}\right)$ were measured using T-DOC air-charged 7F urodynamic catheters (Laborie, Mississauga, ON, Canada). The bladder was filled with normal saline at a filling rate that ranged between 20 and $30 \mathrm{mls}$ per minute. The detrusor pressure was calculated as the difference between the intra-vesical and intra-abdominal pressure $\left(P_{\text {ves }}-P_{\text {abd }}\right)$. Similarly, bladder filling was stopped after $500 \mathrm{mls}$ infusion or if the patient developed urgency, leaking, symptoms of autonomic dysreflexia or a sudden rise of $P_{\text {det }}$ to $>80 \mathrm{~cm} \mathrm{H}_{2} \mathrm{O}$.

\section{Outcome assessment}

All recorded data were interpreted in relation to individual patients' clinical and neurological findings. The terminology used for types of NBD was based on that recommended by the International Continence Society. ${ }^{9}$ Any evidence of involuntary detrusor contraction occurring during the filling phase (spontaneous or provoked) was considered as evidence of neurogenic detrusor overactivity. Neurogenic detrusor underactivity was defined as a contraction of reduced strength and/or duration, resulting in prolonged bladder emptying and/or failure to achieve complete bladder emptying within a normal time span. In the absence of cystometric evidence of detrusor contraction, neurogenic detrusor acontractility was 


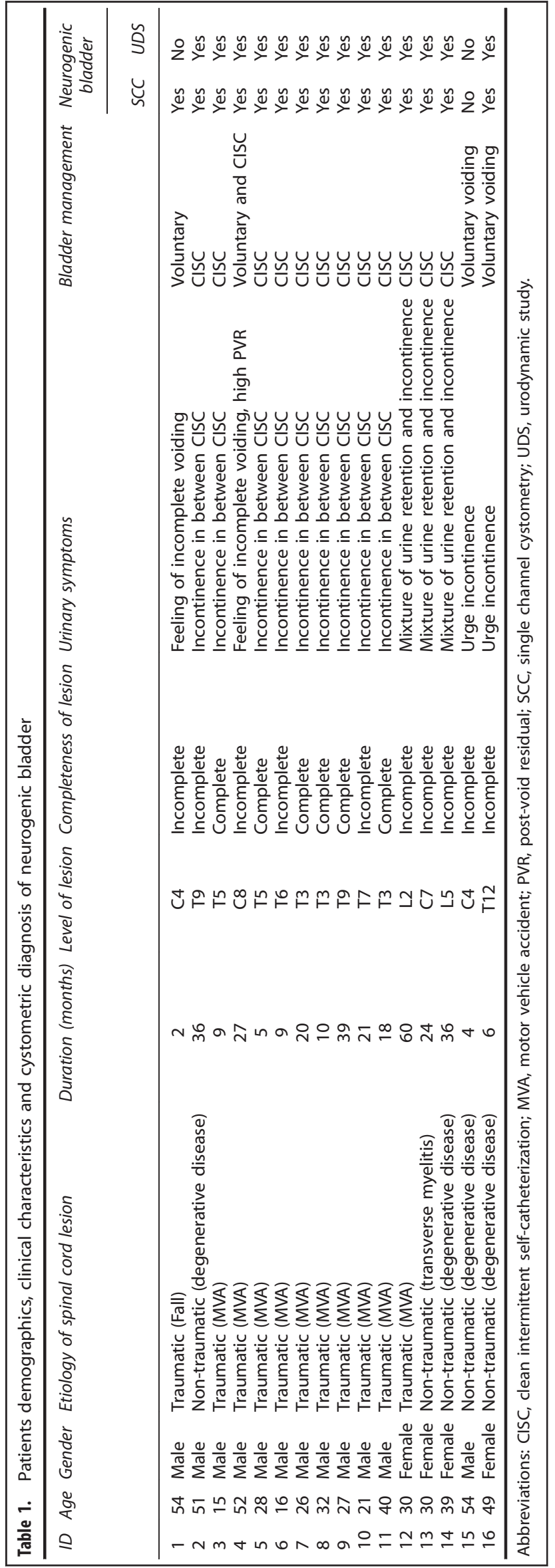

Table 2. Diagnostic test analyses of single channel cystometry compared with urodynamic study

\begin{tabular}{lccc}
\hline Neurogenic bladder diagnosis & \multicolumn{3}{c}{ Urodynamic Study } \\
\cline { 2 - 4 } & Yes & No & Total \\
\hline Single channel cystometry & & & \\
Yes & 14 & 1 & 15 \\
No & 0 & 1 & 1 \\
Total & 14 & 2 & 16 \\
\hline
\end{tabular}

Abbreviation: $\mathrm{Cl}$, confidence interval. Sensitivity $=100 \%(95 \% \mathrm{Cl}$ : $76.84-$ $100.00 \%)$. Specificity $=50 \% \quad(95 \%$ Cl: $1.26-98.74 \%)$. Positive predictive value $=93.3 \%(95 \% \mathrm{Cl}: 68.05-99.83 \%)$. Negative predictive value $=100 \%$ (95\% Cl: $2.50-100.00 \%)$.

diagnosed. Cystometric evidence of involuntary contractions of the external urethral sphincter during involuntary detrusor contraction was diagnosed as neurogenic DSD. This condition can only be suspected clinically during SCC procedures and will warrant further confirmatory test with UDS.

\section{Statistical analyses}

The baseline demographic and clinical features of the patients including age, gender, etiology, duration, level and severity of the $\mathrm{SCl}$ were recorded. All data were analyzed using the SPPS software version 20.0 (IBM Corporation, Armonk, NY, USA). The categorical data were analyzed using descriptive statistics and reported in frequency and percentage. Comparative analyses were carried out for cystometric diagnoses data obtained from SCC and UDS procedures. Then, the sensitivity, specificity, negative predictive value (NPV) and positive predictive value (PPV) were calculated using the Diagnostic Test procedures.

Sensitivity of a clinical test refers to the ability of the test to correctly identify those patients with the disease, whereas specificity refers to the ability of the test to correctly identify those without the disease. PPV refers to the likelihood that a patient has the disease given that the test result is positive, whereas NPV refers to the likelihood that a patient does not have the disease, given that the test result is negative. ${ }^{11}$ In this study the sensitivity, specificity, PPV and NPV of SCC were compared toward the gold standard test, that is, the urodynamics Study.

\section{RESULTS}

A total of 16 patients were studied, of which $75 \%$ (12/16) were male and $25 \%$ were (4/16) female. The mean duration of injury was $20.38 \pm 16.09$ months (range 2-60 months). With regard to neurological category, $25 \%(4 / 16)$ were incomplete tetraplegia, $37.5 \%(6 / 16)$ were incomplete paraplegia and $37.5 \%(6 / 16)$ were complete paraplegia patients. The majority of the patients, $75 \%$ $(12 / 16)$ managed their bladder through intermittent catheterization, $18.75 \%(3 / 16)$ patients are able to void voluntarily, whereas $6.25 \%(1 / 16)$ of patients combined voluntary voiding and clean intermittent self-catheterization techniques. With regard to bladder symptoms $56.25 \%$ (9/16) had reflex incontinence, $12.5 \%$ $(2 / 16)$ had urge incontinence and $18.75 \%(3 / 16)$ of patients had a mixture of urinary retention and incontinence (Table 1).

In $87.5 \%(14 / 16)$ of patients, neurogenic bladder diagnosis was confirmed by both SCC and UDS studies. Diagnostic test evaluation showed that SCC was found to have $100 \%$ sensitivity, 50\% specificity, 93.33\% PPV and 100\% NPV for neurogenic bladder diagnosis (Table 2). No relevant adverse effects were observed when patients were subjected to both procedures.

With regard to specific cystometric bladder diagnosis, all three patients diagnosed with neurogenic detrusor acontractility by SCC had comparable UDS findings. It was also noted that of the 9 patients with neurogenic detrusor overactivity with suspicion of DSD diagnosed via SCC, only $55.55 \%$ (5/9) had comparable UDS findings (Table 3 ). 
Table 3. Type of neurogenic bladder and cystometric parameters between single channel versus urodynamic study

\begin{tabular}{lcc}
\hline Type of neurogenic bladder & SCC (\%) \\
\hline Neurogenic detrusor overactivity with detrusor sphincter dyssinergia & $9(56.25)$ & UDS (\%) \\
Neurogenic detrusor overactivity without detrusor sphincter dyssinergia & $3(18.75)$ & $5(31.2)$ \\
Neurogenic detrusor acontractility & $3(18.75)$ & $3(37.5)$ \\
Normal detrusor function & $312.31 \pm 166.7$ & $3(18.75)$ \\
Maximum cystometric capacity (mls); (mean \pm s.d.) & $33.93 \pm 29.11$ & $344.62 \pm 208.6$ \\
Maximum recorded detrusor pressure at filling (cm $\left.\mathrm{H}_{2} \mathrm{O}\right)$; (mean \pm s.d.) & $60.87 \pm 37.38$ \\
Maximum recorded detrusor pressure at voiding or leaking $\left(\mathrm{cm} \mathrm{H} \mathrm{H}_{2} \mathrm{O}\right) ;($ mean \pm s.d.) & & $30.25 \pm 27.12$ \\
\hline Abbreviations: SCC, single channel cystometry; UDS, urodynamic study. & & $51.12 \pm 28.53$ \\
\hline
\end{tabular}

The mean cystometric capacity with SCC was slightly lower $(312.31 \pm 166.7 \mathrm{mls})$ compared with UDS $(344.62 \pm 208.6 \mathrm{mls})$. It was also noted that both the mean maximum recorded detrusor pressures during filling phase $\left(F P_{\text {detmax }} 33.93 \pm 29.11\right.$ versus 30.25 $+27.12)$ and voiding or leaking phase (VP detmax $60.87 \pm 37.38$ versus $51.12 \pm 28.53)$ were higher with SCC than with UDS (Table 3).

\section{DISCUSSION}

Over the past few decades, the management of NBD in patients with $\mathrm{SCl}$ has improved tremendously especially in centers with UDS availability. However in centers without UDS, it remains challenging as an objective diagnosis of NBD cannot be made.

To our knowledge, this is the first study that compares the diagnostic accuracy of SCC to UDS in patients with SCl. In this pilot study, we found that the diagnosis of neurogenic bladder detected by SCC was comparable in $87.5 \%(14 / 16)$ of patients to confirmatory UDS. This is in relative agreement to previous comparison studies involving bladder dysfunction of nonneurogenic origin. Sutherst et al. ${ }^{5}$ in their study comparing SCC and UDS in diagnosing bladder instability in 100 women with symptoms of urinary incontinence found $93 \%$ of their subjects produced a replicable result. Similarly, Wheeler et al. ${ }^{6}$ in his study involving 30 women with voiding dysfunction reported that a simple cystometry had an accuracy of $93 \%$ in detecting detrusor instability in comparison with results from a four-channel cystometry.

In managing bladder dysfunction following $\mathrm{SCl}$, a comprehensive history, detailed neurological examination, review of bladder diary, urine and blood investigations and nurses' observations are of utmost importance. These data in combination with information on the level and severity of the $\mathrm{SCl}$ will enable clinicians to predict the detrusor and sphincter dysfunction in around $80 \%$ of patients. $^{12}$ This added information could have been the likely reason for the 100\% sensitivity, 93.3\% PPV and 100\% NPV obtained in our study, which is higher than previous comparison studies of non-NBD. Ouslander et al. ${ }^{7}$ reported, out of 110 geriatric patients with urinary incontinence diagnosed with detrusor motor instability or hyperreflexia using multi-channel cystometry, a simple cystometry study has $77 \%$ sensitivity, $79 \%$ specificity and $85 \%$ PPV. In another report by Wall et al. ${ }^{8}$ involving 70 women with urinary incontinence using SCC and cough stress test reported a sensitivity of $64 \%$ for demonstration of urge incontinence and a specificity of $86.8 \%$ for the demonstration of detrusor instability.

It is well known that UDS is more sensitive in detecting changes in detrusor pressure compared with SCC. This is confirmed in a study by Bates et al. ${ }^{13}$ which showed the importance of excluding changes in intra-abdominal pressure as it may influence intrinsic detrusor pressure. Abnormal breathing patterns, spasticity and altered bowel movements which are all common in patients with $\mathrm{SCl}$, could affect detrusor pressure value. This could explain the low specificity of $50 \%$ obtained in this study. The changes in the detrusor pressure reading could have been affected by changes in intra-abdominal pressures. Similar reasoning could also explain the higher recorded mean maximum detrusor pressure reading during both filling $\left(F P_{\text {detmax }}\right)$ and voiding or leaking $\left(V P_{\text {detmax }}\right)$ phase by SCC compared with that of UDS. Thus, to ensure optimum accuracy of SCC, the authors concluded that close observation and monitoring are crucial when performing the procedure. Elevated intra-vesical pressure reading caused by raised intra-abdominal pressure due to obvious reasons such as movement, spasticity or abnormal breathing pattern should be disregarded and not attributed as detrusor contractions. In addition, all data recording, study interpretation and reporting should be done by the person performing the procedure.

It is of interest to note that in our study the mean maximum cystometric capacity was lower with SCC evaluation compared with that of UDS. Though the two studies were not performed in the same session, we hypothesize this could be the effect of repetitive cystometry resulting in the bladder's improved ability to accommodate higher volumes. Broekhuis et al. ${ }^{14}$ in his study involving 152 women with urinary incontinence that underwent standardized UDS which included two filling cystometry and pressure-flow studies in the same session reported a systematically higher maximum cystometric capacity in the second evaluation.

In addition, we note in this study that all patients with neurogenic detrusor acontractility diagnosed using SCC had a comparable diagnosis using UDS. However only about half of those diagnosed with neurogenic detrusor overactivity with DSD suspicion had a comparable diagnosis using UDS. These findings reflected the main limitation of SCC in particular ruling out the presence of DSD. UDS is better equipped to diagnose DSD as additional information especially the external surface sphincter electromyography activity, voiding cystourethrogram and/or urethral pressure profilometry are recorded during the procedure.

DSD is common post-SCl and it has been estimated to affect $75-100 \%$ of patients with suprasacral lesions. ${ }^{2,12,15}$ Serious urological complications such as reduced bladder compliance, elevated upper tract pressures, hydronephrosis, vesicoureteral reflux and even renal failure have been associated with improperly managed DSD. 2,12,15 Thus, the role of UDS is critical as it provides not only a confirmatory diagnosis of DSD, additionally it is also able to monitor the outcome of intervention. ${ }^{15}$ Therefore, the authors recommended that it is mandatory for all patients with clinical or SCC suspicion of having DSD, to have early UDS for confirmation of diagnosis so that proper bladder management strategies are instituted in a timely manner.

\section{CONCLUSION}

In our opinion, SCC when carefully interpreted in relation to clinical findings may be considered a reasonably accurate, practical, safe and cheap method for diagnosing NBD especially in $\mathrm{SCl}$ patients in centers with limited access to UDS. However, we are not able to establish its clinical application for diagnostic 
confirmation of DSD. Thus, UDS should be performed in all SCI patients with a clinical suspicion of DSD.

Limitations of this study

To our knowledge, this is the first study to evaluate the applicability of SCC to diagnose NBD in the SCI population. However, the small sample size is one of our major limitations in the interpretation of the study findings. This was due to limited access to UDS services, which had to be done in another hospital. We plan to repeat this study to re-evaluate SCC diagnostic accuracy once we obtain a UDS machine in our center.

Another major limitation is the inability to accurately confirm the clinical suspicion of DSD. Thus, we are of the opinion that all patients with a clinical suspicion of DSD must be evaluated using UDS for objective confirmation.

\section{Local clinical implication}

The application of SCC had allowed the authors to objectively evaluate NBD in a timely manner. It facilitates our clinical decision on pharmacologic intervention and review of the treatment outcome. For example, early treatment with anticholinergic can be considered in patients with cystometric evidence of neurogenic detrusor overactivity. Exception to this was $\mathrm{SCl}$ patients with clinical suspicion of DSD; they were subjected to early mandatory UDS for diagnosis confirmation and further management.

\section{ACKNOWLEDGEMENTS}

We would like to thank the Director General of Health Malaysia for his permission to publish this paper. We also wish to thank Ms Nadiah Md Sa'at of Clinical Research Centre, Ministry of Health Malaysia for her review of statistical analyses and interpretation.

\section{COMPETING INTERESTS}

The authors declare no conflict of interest.

\section{REFERENCES}

$1 \mathrm{Ku} \mathrm{JH}$. The management of neurogenic bladder and quality of life in spinal cord injury. BJU Int 2006; 98: 739-745.

2 Jeong SJ, Cho SY, Oh SJ. Spinal cord/brain injury and neurogenic bladder. Urol Clin North Am 2010; 37: 537-546.

3 Consortium for Spinal Cord Medicine. Bladder management for adults with spinal cord injury: a clinical practice guideline for healthcare providers. J Spinal Cord Med 2006; 29: 527-573.

4 Stephenson TP, Wein AJ. Urodynamic; Principles Practice and Application. Churchill Livingstone: Edinburgh, Scotland, 1986.

5 Sutherst JR, Brown MC. Comparison of single channel and multichannel cystometry in diagnosing bladder instability. Br Med J (Clin Res Ed) 1984; 228: $1720-1722$.

6 Wheeler JS, Niecestro RM, Fredian C, Walter JS. Comparison of a simple cystometer with a multi-channel cystometer in females with voiding dysfunction. Int Urogynecol J 1991; 2: 90-93.

7 Ouslander J, Leach G, Abelson S, Staskin D, Blaustein J, Raz S. Simple versus multichannel cystometry in the evaluation of bladder function in an incontinent geriatric population. J Urol 1998; 140: 1482-1486.

8 Wall LL, Wiskind AK, Taylor PA. Simple bladder filling with a cough stress test compared with subtracted cystometry for the diagnosis of urinary incontinence. Am J Obstet Gynecol 1994; 171: 1472-1477.

9 Abrams P, Cardozo L, Fall M, Griffiths D, Rosier P, Ulmsten U et al. The standardization of terminology of lower urinary tract function: report from the standardization subcommittee of the International Continence Society. Neurourol Urodyn 2002; 21: 167-178.

10 Wyndaele JJ, THi HV, Pham BC, Kovindha A, Huong VT, Weerts E. The use of onechannel water cystometry in patients with a spinal cord lesion: practicalities, clinical value and limitations for the diagnosis of neurogenic bladder dysfunction. Spinal Cord 2009; 47: 526-530.

11 Lalkhen AG, McCluskey A. Clinical tests: sensitivity and Specificity. Contin Educ Anaesth Crit Care Pain 2008; 8: 221-223.

12 Wyndaele JJ. Correlation between clinical neurological data and urodynamic function in spinal cord injured patients. Spinal Cord 1997; 35: 213-216.

13 Bates CP, Whiteside CG, Turner-Warwick R. Synchronous cine/pressure/flow cystourethrography with special reference to stress and urge incontinence. $\mathrm{Br} J$ Urol 1970; 42: 714-723.

14 Broekhuis SR, Kluivers KB, Hendriks JC, Massolt ET, Groen J, Vierhout ME. Reproducibility of same session repeated cystometry and pressure-flow studies in women with symptom of urinary incontinence. Neurourol Urodyn 2010; 29: 428-431.

15 Bacsu C-D, Chan L, Tse V. Diagnosing detrusor sphincter dyssynergia in the neurological patient. BJU Int 2012; 109 (Suppl S3): 31-34. 